\title{
Clinical spectrum of acute kidney injury among hospitalized patients in coastal districts of Andhra Pradesh
}

\author{
Suresh $\mathbf{V}^{1}$, Gupta NSRC ${ }^{2}$, Bhargavi E. $\mathbf{U}^{3}$ \\ ${ }^{1}$ Dr Vaddadi Suresh, Associate Professor, Department of Medicine, ${ }^{2}$ Dr NSRC Gupta, Assistant Professor, Department of \\ Medicine, ${ }^{3}$ Dr E Usha Bhargavi, Assistant Professor, Department of Pathology; all authors are affiliated with GSL \\ Medical College and Hospital, Rajahmundry, India.
}

Address for Correspondence: Dr Vaddadi Suresh, email: sureshvaddadi@yahoo.co.in

\begin{abstract}
Introduction: Acute kidney injury (AKI) is one of the important overall causes of mortality in hospitalized patients. Variability in definitions of AKI has led to gross variations in the incidence across the world. Multiple metabolic derangements in these patients can increase the mortality and morbidity. Our study aims at studying the incidence, clinical, biochemical, hematological and sonographic abnormalities seen in hospitalized patients with AKI. Methods and Material: Case records of 237 patients admitted in various departments of a tertiary care center over a three year period from 2010 to 2013, who developed AKI were included in the study. The etiologies, various hematological and biochemical test reports, ultrasonography of abdomen and treatment given was noted. Statistical analysis was done to find the pattern of AKI and the prevalence of various biochemical and metabolic abnormalities. Results: The incidence of AKI among hospitalized patients was $1.9 \%$. Out of 237 patients with AKI, $62 \%$ were males. $70 \%$ belonged to medical and allied specialties. The most common cause of AKI was infection related. Fever was the most common symptom (48\%) and common laboratory abnormalities included anemia (70\%), hyperkalemia (36\%) and proteinuria (29\%). Mortality in this study was $21(9 \%), 73 \%$ received conservative therapy while $27 \%$ had undergone hemodialysis. Conclusions: AKI is highly prevalent among hospitalized patients admitted in medical and allied departments. Infections top the list among the causes of AKI. Various metabolic and biochemical abnormalities are seen, most of them can be managed conservatively and up to one third may require hemodialysis.
\end{abstract}

Key-words: Acute Kidney Injury, Anemia, Hemodialysis, Hyperkalemia, Infections.

\section{Introduction}

AKI is becoming more and more prevalent day by day and the burden is expected to reach the levels of pandemic in the years to come. There is a trend to ignore minor rise of creatinine, especially in non specialized centers, and this has lead to gross underestimation of the true incidence of AKI in India. It has been well established that even minor increases in S. Creatine can increase mortality over the long term [1]. Patients who have apparently recovered from AKI at discharge may still be at risk of future development of chronic kidney disease. Recent advances in the classification and diagnostic criteria of AKI including the RIFLE and AKIN criteria have categorized the patients based on Serum Creatinine, urine output and

Manuscript received $14^{\text {th }}$ July 2016

Reviewed: 24 $4^{\text {th }}$ July 2016

Author Corrected: $10^{\text {th }}$ August 2016

Accepted for Publication $22^{\text {nd }}$ August 2016 glomerular filtration rate (GFR) $[2,3]$. They help in early recognition and grading of severity of patients and in assessing the outcome. The list of diseases that can result in AKI is long. Most important are infections, post surgical causes, drug induced, obstructive causes and so on. Importantly, in each disease, the cause of AKI is usually multifactorial [4]. AKI is the most important cause of death in sepsis patients. The burden of infection related AKI is also underestimated.

The major reasons behind it are under reporting of cases and lack of multicentric long term studies addressing the issue, especially in India. Various metabolic, biochemical and hematological abnormalities exist in patients with AKI, most of which can be managed when recognized early. Identification and treatment of the primary cause of AKI is important for good outcome. 


\begin{abstract}
Aims
This is a retrospective study which aims at studying the incidence of AKI and various clinical, metabolic, biochemical, hematological and sonographic abnormalities seen in hospitalized patients who are diagnosed with AKI.
\end{abstract}

Place of study: GSL medical college and general hospital, Rajahmundry.

Type of study: Retrospective observational study.

Subjects and methods: Case records of 12367 patients aged over 12 years, admitted in a tertiary care center over a three year period from 2010 to 2013 were screened. Of these, case sheets of inpatients admitted in general wards, special wards and ICUs of various departments with evidence of acute kidney injury were included in the study.

Inclusion criteria: The selection was based on elevated S.Creatinine more than 1.5 times above the baseline or previous value at any time during hospital stay.
Exclusion criteria: Cases with normal S. Creatinine or with evidence of chronic kidney disease like bilaterally small kidneys, past history of CKD and case records with incomplete documentations were excluded from the study.

\section{Methods}

A detailed analysis regarding the incidence of AKI among admitted patients, age, gender, cause of AKI and the type of treatment given for renal disease was undertaken. Data regarding the investigations done including complete blood picture, S. Creatinine, urea, blood sugars, liver function tests, ultrasonography of abdomen, electrolytes, serum calcium and phosphate and complete urine analysis was collected to the available extent. The prevalence of various abnormalities was noted. The clinical symptoms documented at the time of admission were noted.

Statistics: Microsoft Excel 2007 software is used and the prevalence of various abnormal parameters has been described in the form of percentages.

\section{Results}

Out of 12367 cases, 237 cases have shown evidence of AKI (1.9\%). Males comprised 147 (62\%) cases whereas females comprised 90 cases (38\%). The most common age group was between 40 to 50 years. Out of 237 patients, full reports were available for complete blood picture, S. Creatinine, blood urea, serum electrolytes, random blood sugars and urine analysis. USG abdomen reports were available in 173 cases, Arterial blood gas analysis in 94 cases, liver function tests in 149 cases, serum calcium in 104 and phosphate in 74 cases. The majority of the cases (70\%) belonged to medical and allied departments, followed by surgical and allied departments (30\%). The frequency distribution of various cases of AKI in different departments is shown in Figure-1.

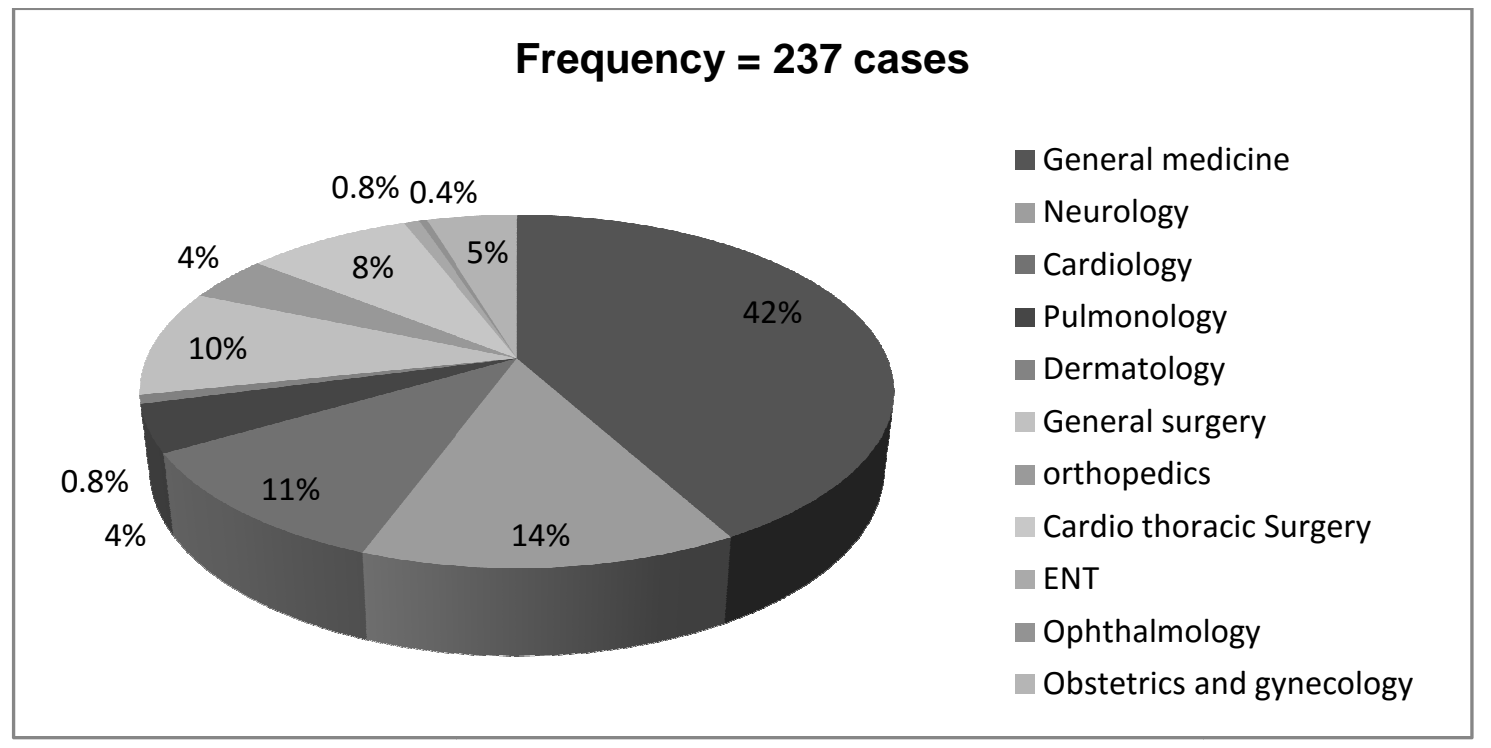

Figure-5: Shows the distribution of AKI cases among medical and surgical departments. 
The clinical presentation of patients during treatment period is shown in Table -1

Table-1: Shows various signs and symptoms in AKI patients.

\begin{tabular}{|c|c|}
\hline Clinical Features & $\mathbf{n = 2 3 7}(\mathbf{\%})$ \\
\hline Fever & $115(48.5 \%)$ \\
\hline Oliguria/anuria & $105(44.3 \%)$ \\
\hline Pallor & $96(40.5 \%)$ \\
\hline Vomitings & $72(30.3 \%)$ \\
\hline Pedal edema & $51(21.5 \%)$ \\
\hline Jaundice & $49(20.6 \%)$ \\
\hline Altered sensorium & $40(16.8 \%)$ \\
\hline Rash & $35(17.4 \%)$ \\
\hline Polyuria & $23(9.7 \%)$ \\
\hline Hiccups & $17(7.2 \%)$ \\
\hline Seizures & $8(3.4 \%)$ \\
\hline
\end{tabular}

The prevalence of various hematologic and biochemical abnormalities is shown in Table -2

Table-2: Shows the prevalence of various abnormalities of cell counts and biochemical derangements.

\begin{tabular}{|ll|c|}
\hline \multicolumn{1}{|c|}{ Hematological and biochemical abnormalities } & Total number $=\mathbf{2 3 7}(\mathbf{\%})$ \\
\hline 1. & Anemia & $165(69.6 \%)$ \\
\hline 2. & Leukocytosis & $111(46.8 \%)$ \\
\hline 3. & Thrombocytopenia & $68(28.6 \%)$ \\
\hline 4. & Hyperkalemia & $85(35.9 \%)$ \\
\hline 5. & Hypokalemia & $56(23.6 \%)$ \\
\hline 6. & Hyperglycemia & $44(18.5 \%)$ \\
\hline 7. & Hyponatremia & $33(13.9 \%)$ \\
\hline 8. & Leukopenia & $14(5.9 \%)$ \\
\hline 9. & Hypernatremia & $9(3.7 \%)$ \\
\hline
\end{tabular}

Among other reports available, metabolic acidosis was found in 51 out of 94 (54.2\%), elevated liver enzymes in 73 out of 149 (49\%), hypocalcemia in 17 out of $104(16.3 \%)$ and hyperphosphatemia in 12 out of 74 cases (16.2\%). The underlying etiology which might be directly or indirectly responsible for AKI is shown in Figure-2.

In 27 cases (12\%), the cause of AKI could not be established. Miscellaneous causes of AKI that were identified included contrast nephropathy, acute glomerulonephritis, acute pancreatitis, drug induced interstitial nephritis, snake bites, rhabdomyolysis etc.

The abnormalities in urinary sediment are presented in Table-3.

Table-3 showing urinary abnormalities.

\begin{tabular}{|c|c|}
\hline Urine Sediment & $\mathbf{n = 2 3 7 ( \% )}$ \\
\hline Albuminuria & $69(29.1 \%)$ \\
\hline Hematuria & $37(15.6 \%)$ \\
\hline WBC casts & $22(9.3 \%))$ \\
\hline Hyaline casts & $21(8.8 \%)$ \\
\hline Pus cell casts & $17(7.2 \%)$ \\
\hline RBC casts & $13(5.4 \%)$ \\
\hline Eosinophiluria & $7(2.9 \%)$ \\
\hline
\end{tabular}


Ultrasonography of abdomen reports available in 173 patients revealed abnormalities as shown in Table - 4

Table-4 shows the report of Ultrasonography of abdomen.

\begin{tabular}{|c|c|}
\hline Ultrasonography Of Abdomen & $\mathbf{N = 1 7 3}(\mathbf{\%})$ \\
\hline Renal parenchymal changes & $124(71.6 \%)$ \\
\hline Hepato/splenomegaly & $55(31.7 \%)$ \\
\hline Ascites & $43(24.8 \%)$ \\
\hline Non obstructive calculi & $22(12.7 \%)$ \\
\hline Cystitis & $20(11.5 \%)$ \\
\hline Obstructive Calculi (Hydronephrosis) & $18(10.4 \%)$ \\
\hline Pyelonephritis & $11(6.3 \%)$ \\
\hline Tumors & $10(5.8 \%)$ \\
\hline Peritonitis & $9(5.2 \%)$ \\
\hline Gravid uterus & $7(4 \%)$ \\
\hline Pyometra & $2(1.1 \%)$ \\
\hline Pancreatitis & $2(1.1 \%)$ \\
\hline
\end{tabular}

64 Patients (27\%) underwent hemodialysis as per indications and others were managed conservatively (73\%). The mortality in our study is $21(9 \%)$ and in many cases the mortality was attributed to the primary cause. Most of the patients recovered from AKI during the hospital stay.

\section{Discussion}

Our hospital is a multidisciplinary tertiary care centre, where patients from adjacent five districts visit. Most of the cases of AKI are infection related especially malaria, as many endemic areas of malaria are located around $100 \mathrm{~km}$ radius. Many studies have evaluated the AKI incidence and prognosis among ICU patients both in India and overseas. The actual burden of AKI extends beyond ICUs and many stable patients in wards can develop AKI. Above all, population based community surveys to estimate the prevalence of AKI are truly limited.

The incidences varied from study to study and were reported to be between 140-600 per million population [5]. The incidence of AKI in our study (1.9\%) is similar to the data from US with a reported incidence ranging from $1-7 \%$ of all hospitalized patients [6,7]. AKI in ICU patients has a reported incidence of $20-50 \%$ [8]. Globally, AKI is more common in males and our study showed a clear male preponderance and higher incidence among men over 50 years of age. Falling renal reserve is likely to result in kidney damage when exposed to infections or toxins.

Most of the patients in our study were from medical and allied specialities. This relies on the fact that the most common causes of AKI are infections and nephrotoxins, both are more likely to be seen in medical wards. In our study, patients with cerebrovascular accidents had a high incidence of AKI especially pre renal AKI, and most of these cases quickly recovered within few days. This might be due to dehydration induced by drugs like mannitol and underlying common risk factor like diabetes. In our study, infections, especially Malaria, Leptospirosis and Urinary tract infections causing gram negative sepsis formed majority of medical cases. This is consistent with some of the studies focusing on malaria as a very important cause of AKI both in the wards and in ICUS in south India [9]. Contrast nephropathy is another important medical cause of AKI, especially in diabetics.

Patients may develop transient azotemia following contrast injection that can be managed by good hydration, but at times patients may need renal replacement therapy. Among surgical departments, post surgical AKI, especially after cardiac bypass is extremely important. The rate of AKI after elective cardiac surgery ranges from $15-30 \%$ but the mortality due to renal disease is low [10]. The most consistent factor with proven association with AKI is the duration of bypass with rates being higher with prolonged surgeries commonly involving valves [11].

Fever was the most common clinical feature associated with AKI in our study which may be explained by infection. Oliguria was seen in half and polyuria in one fourth of the patients. Patients in the recovery phase of 
AKI can develop polyuria, which makes them susceptible to electrolyte depletion. Vomitings, altered sensorium, hiccups and seizures could represent uremic symptoms which should alert physicians to consider the need for RRT. Anemia was seen in $70 \%$ of cases. Similar finding was observed in a recent study from Iran showing very high prevalence of anemia in AKI patients [12,13]. Anemia is a known complication of chronic kidney disease, but the association of anemia with AKI is poorly understood. The cause could be multifactorial including poor nutrition, reduced appetite along with the primary cause of AKI.

Leukocytosis is probably due to bacterial and malarial infections and leukopenia and thrombocytopenia were attributed to viral infections. Hyperkalemia was the most common electrolyte disturbance and hypernatremia was least common. Hyperkalemia is due to reduced renal clearence of potassium due to tubular damage and metabolic acidosis. Hypernatremia in the setting of AKI suggests severe intravascular volume depletion and is a sign of poor hydration. Many cases where cause could not be identified were regarded as pre renal as S.Creatinine got corrected quickly with hydration before etiology was established. Proteinuria was the most common urinary abnormality in our study.

In AKI, proteinuria is due to glomerular damage leading to leaking of albumen and globulin or tubular injury leading to excretion of globulin. RBC casts suggest glomerulonephritis, WBC casts suggest tubulointestitial nephritis and drug induced AKI while granular casts are seen in cases with established renal injury. Eosinophiluria is seen in acute Intestitial nephritis, commonly drug induced, though not specific [14].

USG abdomen is a very important tool especially in ruling out obstructive causes and in determining the extent of renal cortical loss. Bilateral contracted kidneys are good indicators of CKD rather than AKI. Many patients of AKI show altered echo pattern of kidneys and loss of corticomedullary differentiation indicates significant nephron damage. In our study, USG abdomen helped in the diagnosis of the cause of AKI in nearly one third in whom USG was done, mostly by revealing obstructive causes like tumors and calculi. $27 \%$ of patients had undergone hemodialysis for different indications like uremic symptoms, refractory hyperkalemia and metabolic acidosis, volume overload etc. Others were managed conservatively with IV fluids, urine output monitoring and management of the primary cause. The mortality of $9 \%$ was mostly attributed to the primary cause like shock, severe malaria, hepatic failure, CVA and surgical causes like peritonitis and tumors. In most of them, S.Creatinine showed a stable pattern till the time of death. In those who received hemodialysis, three died due to complications of uremia. In the previous studies done in ICU patients and overall hospitalized patients, the mortality rates varied widely from $9 \%$ to $72 \%$ when conducted in mixed population of patients $[15,16]$. The mortality in our study is less compared to previous studies.

There was no follow up of patients and hence only in hospital mortality was measured. Secondly, patients with AKI due to treatable conditions like severe malaria and leptospirosis formed majority of cases in our study, which might have contributed to reduced mortality compared to other studies. The limitations of our study are non inclusion of urine output to identify AKI which would have picked up more cases, unreliability of records as a measure of disease burden and lack of follow up of patients.

\section{Conclusion}

AKI is highly prevalent among hospitalized patients with various diseases, especially males. Medicine and allied specialities hold most of the cases of AKI. Infections top the list among causes of AKI, and hence early recognition and treatment is warranted. Anemia, hyperkalemia, albuminuria and altered echotexure of kidneys are commonly seen among these patients. Two third of the patients recover with treatment of the primary cause and AKI can be managed conservatively while up to one third of patients may require hemodialysis.

Funding: Nil, Conflict of interest: None initiated, Permission from IRB: Yes

\section{References}

1. Chertow GM, Burdick E, Honour M, Bonventre JV, Bates DW. Acute kidney injury, mortality, length of stay, and costs in hospitalized patients. Journal of the American Society of Nephrology. 2005 Nov $1 ; 16(11): 3365-70$.

2. Bellomo R, Ronco C, Kellum JA, Mehta RL, Palevsky P. Acute renal failure-definition, outcome measures, animal models, fluid therapy and information technology needs: the Second International Consensus Conference of the Acute Dialysis Quality Initiative (ADQI) Group. Critical care. 2004 May 24;8(4):1. 
3. Mehta RL, Kellum JA, Shah SV, Molitoris BA, Ronco C, Warnock DG, Levin A. Acute Kidney Injury Network: report of an initiative to improve outcomes in acute kidney injury. Critical care. 2007 Mar 1;11(2):1.

4. Liano F, Junco E, Pascual J, Madero R, Verde E. The spectrum of acute renal failure in the intensive care unit compared with that seen in other settings. The Madrid Acute Renal Failure Study Group. Kidney international. Supplement. 1998 May;66:S16-24.

5. Liano F, Pascual J, Madrid Acute Renal Failure Study Group. Epidemiology of acute renal failure: a prospective, multicenter, community-based study. Kidney international. 1996 Sep 30;50(3):811-8.

6. Nash K, Hafeez A, Hou S. Hospital-acquired renal insufficiency. American Journal of Kidney Diseases. 2002 May 31;39(5):930-6.

7. Kaufman J, Dhakal M, Patel B, Hamburger R. Community-acquired acute renal failure. American journal of kidney diseases. 1991 Feb 28;17(2):191-8.

8. Case J, Khan S, Khalid R, Khan A. Epidemiology of acute kidney injury in the intensive care unit. Critical care research and practice. 2013 Mar 21;2013.

9. Kute VB, Shah PR, Munjappa BC, Gumber MR, Patel HV, Jain SH, Engineer DP, Naresh VS, Vanikar $\mathrm{AV}$, Trivedi HL. Outcome and prognostic factors of malaria-associated acute kidney injury requiring hemodialysis: A single center experience. Indian journal of nephrology. 2012 Jan;22(1):33.

10. Machado MD, Miranda RC, Takakura IT, Palmegiani E, Santos CA, Oliveira MA, Mouco OM, Hernandes ME, Lemos MA, Maia LN. Acute kidney injury after on-pump coronary artery bypass graft surgery. Arquivos brasileiros de cardiologia. 2009 Sep;93(3):247-52.

11. Englberger L, Suri RM, Connolly HM, Li Z, Abel MD, Greason KL, Dearani JA, Schaff HV. Increased risk of acute kidney injury in patients undergoing tricuspid valve surgery. European Journal of CardioThoracic Surgery. 2012 Sep 22:ezs515.

12. Dorgalaleh A, Mahmudi M, Tabibian S, Khatib ZK, Tamaddon GH, Moghaddam ES, Bamedi T, Alizadeh S, Moradi E. Anemia and thrombocytopenia in acute and chronic renal failure. International journal of hematology-oncology and stem cell research. 2013;7(4):34.

13. Hales M, Solez K, Kjellstrand C. The anemia of acute renal failure: association with oliguria and elevated blood urea. Renal failure. 1994 Jan $1 ; 16(1): 125-31$.

14. Shibasaki T, Ishimoto F, Sakai O, Joh K, Aizawa S. Clinical characterization of drug-induced allergic nephritis. American journal of nephrology. 1991 Jul 1;11(3):174-80.

15. Hoste EA, Clermont G, Kersten A, Venkataraman R, Angus DC, De Bacquer D, Kellum JA. RIFLE criteria for acute kidney injury are associated with hospital mortality in critically ill patients: a cohort analysis. Critical care. 2006 May 12;10(3):1.

16. Samimagham HR, Kheirkhah S, Haghighi A, Najmi Z. Acute kidney injury in intensive care unit: incidence, risk factors and mortality rate. Saudi Journal of Kidney Diseases and Transplantation. 2011 May 1;22(3):464.

\section{How to cite this article?}

Suresh V, Gupta NSRC, Bhargavi E. U. Clinical spectrum of acute kidney injury among hospitalized patients in coastal districts of Andhra Pradesh. Int J Med Res Rev 2016;4(8):1365-1370.doi:10.17511/ijmrr.2016.i08.14. 